\title{
EXTENSION OF THE BIVARIATE CHARACTERIZATION FOR STOCHASTIC ORDERS
}

\author{
RHONDA RIGHTER, * Santa Clara University \\ J. GEORGE SHANTHIKUMAR, ${ }^{* *}$ University of California, Berkeley
}

\begin{abstract}
The bivariate characterization of stochastic ordering relations given by Shanthikumar and Yao (1991) is based on collections of bivariate functions $g(x, y)$, where $g(x, y)$ and $g(y, x)$ satisfy certain properties. We give an alternate characterization based on collections of pairs of bivariate functions, $g_{1}(x, y)$ and $g_{2}(x, y)$, satisfying certain properties. This characterization allows us to extend results for single machine scheduling of jobs that are identical except for their processing times, to jobs that may have different costs associated with them.
\end{abstract}

\begin{abstract}
LIKELIHOOD RATIO ORDERING; HAZARD RATE ORDERING; STOCHASTIC SCHEDULING
\end{abstract}

AMS 1991 SUBJECT CLASSIFICATION: PRIMARY 60E05

SECONDARY 90B35

\section{Preliminaries}

For convenience we list the following results for the bivariate characterization of likelihood ratio, hazard rate, and stochastically ordered random variables (Shanthikumar and Yao (1991)). Throughout we assume $X$ and $Y$ are independent random variables. For any bivariate function, $g(x, y)$, define $\Delta g(x, y):=g(x, y)-g(y, x)$. Also define

$\mathscr{G}_{\mathrm{I}}:=\{g(x, y): g(x, y) \geqq g(y, x) \forall x \geqq y\}$,

$\mathscr{G}_{\mathrm{hr}}:=\{g(x, y): \Delta g(x, y)$ is increasing in $x \forall x \geqq y\}$,

$\mathscr{G}_{\mathrm{st}}:=\{g(x, y): \Delta g(x, y)$ is increasing in $x \forall x\}$.

Lemma $1 . X \geqq_{a} Y \Leftrightarrow E[g(X, Y)] \geqq E[g(Y, X)] \forall g \in \mathscr{G}_{\mathrm{a}}$, for a = lr, hr, st respectively.

\section{Main result}

Let $g_{1}(x, y)$ and $g_{2}(x, y)$ be two bivariate functions, and let $\Delta g_{12}(x, y)=g_{1}(x, y)-g_{2}(x, y)$. We consider the following set of conditions on $g_{1}$ and $g_{2}$ :

(a) $\Delta g_{12}(x, y) \geqq-\Delta g_{12}(y, x)$, i.e. $g_{1}(x, y)-g_{2}(x, y) \geqq g_{2}(y, x)-g_{1}(y, x)$, for all $x \geqq y$.

(b) $\Delta g_{12}(x, y) \geqq 0$, i.e. $g_{1}(x, y) \geqq g_{2}(x, y)$ for all $x \geqq y$.

(c) $g_{1}(x, y) \geqq g_{2}(y, x)$ for all $x \geqq y$.

(d) $g_{1}(y, x) \geqq g_{2}(x, y)$ for all $x \geqq y$.

(e) $g_{1}(x, y)$ increasing in $x$ for all $x \geqq y$.

(f) $g_{1}(x, y)$ decreasing in $y$ for all $y \leqq x$.

(g) $\Delta g_{12}(x, y)$ increasing in $x$ for all $x \geqq y$.

(h) $\Delta g_{12}(x, y)$ decreasing in $y$ for all $y \leqq x$.

Received 14 October 1991; revision received 17 December 1991.

* Postal address: Department of Decision and Information Sciences, Santa Clara University, Santa Clara, CA 95053, USA.

** Postal address: Walter A. Haas School of Business, University of California, Berkeley, CA 94720, USA.

Supported in part by NSF grant ECS-8811234. 
Theorem 1 .

(i) $X \geqq_{1 \mathrm{r}} Y \Leftrightarrow E\left[g_{1}(X, Y)\right] \geqq E\left[g_{2}(X, Y)\right]$ for all $g_{1}, g_{2}$ satisfying conditions (a) and (b).

(ii) $X \geqq_{1 \mathrm{r}} Y \Leftrightarrow g_{1}(X, Y) \geqq_{\mathrm{icx}} g_{2}(X, Y)$ for all $g_{1}, g_{2}$ satisfying conditions (a), (b), and (c).

(iii) $X \geqq_{\mathrm{lr}} Y \Leftrightarrow g_{1}(X, Y) \geqq_{\mathrm{st}} g_{2}(X, Y)$ for all $g_{1}, g_{2}$ satisfying conditions (b), (c), and (d) (and therefore (a)).

(iv) $X \geqq_{\mathrm{hr}} Y \Leftrightarrow E\left[g_{1}(X, Y)\right] \geqq E\left[g_{2}(X, Y)\right]$ for all $g_{1}, g_{2}$ satisfying conditions (a) and (g).

(v) $X \geqq_{\mathrm{hr}} Y \Leftrightarrow g_{1}(X, Y) \geqq_{\mathrm{icx}} g_{2}(X, Y)$ for all $g_{1}, g_{2}$ satisfying conditions (a), (b), (c), (e), and (g). (h).

(vi) $X \geqq_{\text {st }} Y \Leftrightarrow E\left[g_{1}(X, Y)\right] \geqq E\left[g_{2}(X, Y)\right]$ for all $g_{1}, g_{2}$ satisfying conditions (a), (g), and

(vii) $X \geqq_{\mathrm{st}} Y \Leftrightarrow g_{1}(X, Y) \geqq_{\mathrm{icx}} g_{2}(X, Y)$ for all $g_{1}, g_{2}$ satisfying conditions (a), (b), (c), (e), (f), (g), and (h).

Proof.

$\Rightarrow$ : For simplicity, let us assume $X$ and $Y$ are continuous random variables, with densities $f_{X}$ and $f_{Y}$ respectively. Then,

$$
\begin{aligned}
\boldsymbol{E}\left[\Delta g_{12}(X, Y)\right]= & \int_{y} \int_{x}\left[g_{1}(x, y)-g_{2}(x, y)\right] f_{X}(x) f_{Y}(y) d x d y \\
= & \int_{y} \int_{x \geqq y}\left\{\left[g_{1}(x, y)-g_{2}(x, y)\right] f_{X}(x) f_{Y}(y)\right. \\
& \left.-\left[g_{2}(y, x)-g_{1}(y, x)\right] f_{X}(y) f_{Y}(x)\right\} d x d y \\
\geqq & \int_{y} \int_{x \geqq y}\left[g_{1}(x, y)-g_{2}(x, y)\right]\left[f_{X}(x) f_{Y}(y)-f_{X}(y) f_{Y}(x)\right] d x d y=: L B
\end{aligned}
$$

where the inequality follows from (a), which holds in all cases.

(i): From (b) and the fact that $X \geqq_{1 \mathrm{r}} Y$, we have $L B \geqq 0$, and therefore $E\left[\Delta g_{12}(X, Y)\right] \geqq 0$.

(iv) and (vi): The proof goes through as in Shanthikumar and Yao (1991), Theorems 3.4 and 4.3 respectively, using the fact that $E\left[\Delta g_{12}(X, Y)\right] \geqq L B$.

(ii) It is easy to check that if $g_{1}$ and $g_{2}$ satisfy conditions (a), (b), and (c), and $h$ is any increasing convex function, then $h\left(g_{1}(x, y)\right)$ and $h\left(g_{2}(x, y)\right)$ satisfy (a) and (b), and therefore, from (i), $E\left[h\left(g_{1}(X, Y)\right)\right] \geqq E\left[h\left(g_{2}(X, Y)\right)\right]$.

(iii), (v) and (vii): The proof is similar to that of (ii).

$\Leftarrow$ : Let $g_{2}(x, y)=g_{1}(y, x)$. then (a), (c), and (d) hold with equality. That (b) holds is equivalent to $g_{1} \in \mathscr{G}_{\mathrm{r}}$, that $(\mathrm{g})$ holds is equivalent to $g_{1} \in \mathscr{G}_{\mathrm{hr}}$, and that $(\mathrm{g})$ and $(\mathrm{h})$ hold is equivalent to $g_{1} \in \mathscr{G}_{\text {st. }}$. The result then follows from Lemma 1 .

\section{Scheduling application}

Consider the following scheduling problem. There are $n$ jobs to be scheduled on a single machine to minimize the total cost, $T C=\sum_{i=1}^{n} f_{i}\left(C_{i}\right)$, where $C_{i}$ is the completion time of job $i$, and $f_{i}$ is its cost function. We say that $f_{i}$ is steeper than $f_{i}, f_{i} \geqq_{\mathrm{s}} f_{j}, f_{i}-f_{j}$ is non-decreasing. We assume the cost functions and processing times are agreeable in the sense that if $E X_{i} \leqq E X_{j}$ then $f_{i} \geqq_{\mathrm{s}} f_{i}$ for all $i$ and $j$, where $X_{i}$ is the processing time for job $i$. For example, the total cost might be the weighted flowtime, with agreeable weights. Then we have the following, where SEPT (LEPT) means shortest- (longest-) processing-time-first.

Theorem 2.

(i) If the processing times are likelihood-ratio ordered, and $f_{i}$ is increasing for all $i$, then the total cost is stochastically minimized (maximized) by SEPT (LEPT).

(ii) If the processing times are hazard-rate ordered, and $f_{i}$ is increasing for all $i$, then the total cost is minimized (maximized) in the increasing convex sense by SEPT (LEPT).

(iii) If the processing times are stochastically ordered, and $f_{i}$ is increasing and concave for all $i$, then the expected total cost is minimized (maximized) by SEPT (LEPT). 
Proof. Suppose policy $\pi$ schedules job $j$ immediately following job $i$, where $\boldsymbol{E} \boldsymbol{X}_{i} \geqq E X_{j}$. Let $\pi^{\prime}$ be the same as $\pi$ except for interchanging jobs $i$ and $j$. We condition on the processing times of all the other jobs besides the interchanged jobs. Then the total costs under policies $\pi$ and $\pi^{\prime}$ are

$$
\begin{aligned}
& T C_{\pi}:=z+f_{i}\left(w+X_{i}\right)-f_{j}\left(w+X_{i}+X_{j}\right)+h\left(X_{i}+X_{j}\right)=: g_{1}\left(X_{i}, X_{j}\right), \\
& T C_{\pi^{\prime}}:=z+f_{j}\left(w+X_{j}\right)+f_{i}\left(w+X_{i}+X_{j}\right)+h\left(X_{i}+X_{j}\right)=: g_{2}\left(X_{i}, X_{j}\right),
\end{aligned}
$$

where $z$ is the total cost of the jobs scheduled before job $i(j)$ under $\pi\left(\pi^{\prime}\right), w$ is the completion time of the job scheduled immediately before job $i(j)$ under $\pi\left(\pi^{\prime}\right)$, and $h\left(X_{i}+X_{j}\right)$ is the total cost of the jobs scheduled after job $j(i)$ under $\pi\left(\pi^{\prime}\right)$. Then one can easily check that $f_{i} \leqq_{\mathrm{s}} f_{j}$ and $f_{l}$ increasing for all $l$ implies that conditions (a), (b), (c), (d), (e), and (g) hold (but not (f)), and that if $f_{l}$ is also concave for all $l$, then condition (h) holds as well. Thus, $T C_{\pi^{\prime}}$ will be no greater than $T C_{\pi}$ in the appropriate sense by Theorem 1 . The result follows using successive interchanges.

It is also possible to show Theorem 2 using the approach of Chang and Yao (1990) by using arrangement-increasing functions instead of symmetric functions (Chang, personal communication, 1991). See also Frenk (1991a, b) for related work with weaker ordering relations.

\section{Acknowledgement}

We are grateful to Richard Weber for pointing out an error in the original conditions for (v) and (vii) in Theorem 1.

\section{References}

ChANG, C. S. AND YAO, D. D. (1990) Rearrangement, majorization and stochastic scheduling. Preprint.

FRENK J. B. G. (1991a) A general framework for stochastic one-machine scheduling problems with zero release times and no partial ordering. Prob. Eng. Inf. Sci. 5, 297-315.

Frenk, J. B. G. (1991b) A note on one-machine scheduling problems with imperfect information. Prob. Eng. Inf. Sci. 5, 317-331.

ShanthikumaR, J. G. AND YaO, D. D. (1991) Bivariate characterization of some stochastic order relations. Adv. Appl. Prob. 23, 642-659. 